\title{
Non-linear Dynamics of Chromosome Condensation Induced by Colcemid
}

\author{
Thiago de Salazar e Fernandes ${ }^{1,2 *}$, Isvânia Maria Serafim da Silva ${ }^{2}$, Renato Barros \\ Moraes $^{2}$, Leandro Álvaro de Alcântara Aguiar ${ }^{2}$, Ademir Amaral ${ }^{1}$, Catão Temístocles \\ Barbosa $^{3}$ and Romildo de Albuquerque Nogueira ${ }^{2}$ \\ ${ }^{1}$ Grupo de Estudos em Radioproteção e Radioecologia; Departamento de Energia Nuclear; Av. Prof. Luiz Freire, \\ 1000; 50740-540; Recife - PE - Brasil. ${ }^{2}$ Grupo de Biofísica Teórica e Computacional; Departamento de Morfologia \\ e Fisiologia Animal; Rua Dom Manuel de Medeiros, s/n; 52171-900; Dois Irmãos; Recife - PE - Brasil. ${ }^{3}$ Centro de \\ Informática; Universidade Federal de Pernambuco; 50670-901; Recife - PE - Brasil
}

\begin{abstract}
This study investigated the dynamical process of chromosome condensation after colcemid treatment. Two pairs of human chromosomes, \#2 and \#3, were highlighted for the accurate identification by fluorescence in situ hybridization (FISH). A computerized image analysis system was used to measure the lengths of the two pairs of chromosomes averaged over 50 metaphases of different cultures with colcemid $(0.5 \mu \mathrm{g} / \mathrm{mL})$ added either at 3 or 48 $h$ of a total $72 \mathrm{~h}$ culture period. For determining whether the process of chromosome condensation was chaotic or random, the algorithm of Detrended Fluctuation Analysis (DFA) was used. In order to evaluate the power of the method, the data were shuffled and DFA was performed again. It was found that colcemid prolonged treatment induced a significantly greater chromosome condensation $(p<0.05)$, and the dynamics of this process was determined by the DFA and showed to be chaotic, with scaling exponents with range values $0.5<\alpha \leq 1.0$. When the data were shuffled, the scaling exponent $\alpha$ reduced around to 0.5 , which was characteristic of random events. These findings reinforced the idea that colcemid could interfere in some manner with the structure of chromosomes and the dynamics of chromosome condensation was non-linear.
\end{abstract}

Key words: Non-linear dynamics, chaos, chromosome condensation, colcemid

\section{INTRODUCTION}

Many biological processes have been demonstrated to be non-linear, such as genomic and intergenic regions and heartbeats intervals (Peng et al. 1994; Peng et al. 1995), or the industrial processes such as alcoholic fermentation (Meleiro et al. 2005). This is a field of research called as non-linear dynamics and the analyses of such non-linear processes disclose the fractal properties of these phenomena, such as scaling, self-similarity and fractal dimension (Liebovitch
1998; Stam 2005; Kunicki et al. 2009). Selfsimilarity means that the parts of an object or process resemble to the whole object or process; scaling or dependence on the scale means that the measured properties depend on the scale at which they are realized and the fractal dimension provides a quantitative description of the irregularity of the fractal objects or processes (Liebovitch 1998; Kunicki et al. 2009).

It is well known that DNA has the property of selfreplication and it is the sequence of nitrogen bases purine ( $\mathrm{A}$ and $\mathrm{G}$ ) and pyrimidine ( $\mathrm{T}$ and $\mathrm{C}$ ), which

*Author for correspondence: thiagosalazar@ hotmail.com 
probably generates the feature of fractals. The sequence of bases in DNA follows a non-linear process with long-range correlation if the sequence is coding region, or it is similar to a random walk if it is non-conding region (Peng et al. 1994; Stanley et al. 1996; Havlin et al. 1999; Wang et al. 2008).

Chromatin is a combination of DNA and proteins that makes up the spatial arrangement of genomes within the nucleus. The structure of chromatin is related to its function in a dynamic way. If the region is rich in the genes, it assumes a less condensed configuration, known as euchromatin. Heterochromatin is a less transcriptionally active region, and therefore, more condensed. In eukaryotes, DNA is wrapped around the nucleosomes of $\sim 10 \mathrm{~nm}$ in size. How and whether further compaction into the $30-\mathrm{nm}$ chromatin fiber occurs in vivo is not well known (Zimmer and Fabre 2010).

The fractal properties of the nuclear chromatin, known as "fractal globule" model, have been suggested in contrast to the previous model of "equilibrium globule" (Lieberman-Aiden et al. 2009). In the fractal globule model, the nuclear chromatin crumples into a series of tiny globules like "beads-on-a-string" design. These beads act as monomers in subsequent levels of spontaneous crumpling until only a single globule-of-globules remain. The resulting structure resembles a Peano curve, a continuous fractal trajectory filling densely a 3D space without crossing knots on itself. This model is very attractive for explaining the structure of chromatin segments because they lack knots and would facilitate unfolding and refolding, a crucial step for gene activation, gene suppression, or the cell cycle. In a fractal globule, contiguous regions of the genome tend to form the spatial territories whose size corresponds to the length of the original region. On the other hand, in the equilibrium globule model, the nuclear chromatin is modeled as a compact densely knotted configuration originally used to describe a polymer in a poor solvent at equilibrium (Lieberman-Aiden et al. 2009). This started an emerging interest of understanding how the chromatin is organized within the nucleus and the dynamics of this process ( $\mathrm{Lu}$ et al. 1998; Lieberman-Aiden et al. 2009).

Highly transcribed genes and genes co-regulated during the cell cycle tend to associate one with another when activated (Tanizawa et al. 2010). Certain groups of chromosomes tend to be close together, and their centromeres and telomeres tend to occupy the neighboring regions. However, the principles and forces underlying the nonrandom spatial organization of chromatin are mostly unknown (Zimmer and Fabre 2010).

An increased nuclear chromatin fractal dimension was demonstrated to be associated with more aggressive melanomas, expressing a higher complexity of the chromatin architecture. This fractal parameter has been used as a promising model for the prognosis of malignant melanomas (Bendin et al. 2010). The fractal texture features were also used as the basis for an accurate cytological diagnosis of breast cancer (Einstein et al. 1998). Knowing that the DNA molecule folds in chromosomes in a non-linear manner, it is rational to use non-liner methods to describe the dynamic of this process (Lu et al. 1998). Previous study has demonstrated that the dynamical process of chromosome condensation is apparently random but in fact is a deterministic chaotic event. Fractal geometry refers to the description of structures with self-similarity in any scales (Liebovitch 1998; Stam 2005; Kunicki et al. 2009). In a previous work, Lu et al. (1998) demonstrated that during the transitional stage of chromosomes from premetaphase to early-metaphase, the length changes of chromosomes were chaotic, therefore, presented fractal properties. One of the methods to determine if the process was deterministic chaotic or random, the algorithm was first described by Peng et al. (1994), called Detrended Fluctuation Analysis (DFA). At first, the time series is integrated, and then it is divided into boxes of equal length, $n$. In each box of length $n$, a least-square line is fitted to the data (representing the trend in that box). An algorithm is performed to obtain the relationship of the average fluctuation as a function of box size $\mathrm{n}$, in a double log graphic, in which a linear relationship indicates the presence of scaling exponent (Stam 2005).

The commonly applied technique to study the chromosomes is to accumulate the cells in the metaphase by the addition of the spindle inhibitor colchicine to the cultures for the final $3 \mathrm{~h}$ of $48 \mathrm{~h}$ cell cultures (Edwards et al. 2005). This alkaloid, extracted from the autumn crocus (Colchicum autumnale), destabilizes the microtubules, causing its depolymerization. This process arrests the cells in metaphase (Schwartz 2006). Some researchers have added colcemid, the synthetic analog of colchicine, earlier in order to prevent all the cells progressing into a second cell cycle (Sasaki et al. 
1989; Hayata et al. 1992; Kanda et al. 1994; Fernandes et al. 2008). This is a very important method when the purpose is to score the chromosome aberrations in irradiated lymphocytes from the human peripheral blood (Fernandes et al. 2008; Amaral et al. 2008). On the other hand, one of the drawbacks with this method is that it induces highly condensed chromosomes (Kanda et al. 1994; Fernandes et al. 2008).

This study examined the length of two pairs of chromosomes, namely \#2 and \#3, commonly used for the fluorescence in situ hybridization (FISH) painting, in order to investigate the extent of its over-condensation and the dynamics of length changes related to the extended presence of colcemid in cell cultures, using the algorithm of Peng et al. (1994; 1995) and Lu et al. (1998).

\section{MATERIAL AND METHODS}

\section{Cell Culture}

Blood was donated with informed consent and according to the institutional ethics procedures by a healthy 28 year old male and replicate whole blood cultures were set up by a standard method described by the International Atomic Energy Agency manual (IAEA 2001) with modifications. In brief, $0.3 \mathrm{~mL}$ of the blood was added to a mixture of $4.0 \mathrm{~mL}$ Minimum Essential Medium Eagle - MEM Media, $1.0 \mathrm{~mL}$ of the fetal bovine serum and $0.1 \mathrm{~mL}$ of phytohaemagglutinin (Gibco). The cultures were placed in a $5 \% \mathrm{CO}_{2}$ atmosphere at $37^{\circ} \mathrm{C}$ in an incubator. A solution of, $0.1 \mathrm{~mL}$ of colcemid at a final culture concentration of $0.5 \mu \mathrm{g} / \mathrm{mL}$ was added to the cultures at either 3 or $48 \mathrm{~h}$ before the cultures were terminated at $72 \mathrm{~h}$. Each culture was transferred to a centrifuge tube and spun at $300 \mathrm{x} g$ for 5 minutes. The supernatant was removed and the cell biomass was resuspended in $6.0 \mathrm{~mL}$ of $37{ }^{\circ} \mathrm{C}$ hypotonic $\mathrm{KCl}$ solution and held for $7 \mathrm{~min}$. The tubes were centrifuged again and the cells were re-suspended in $6.0 \mathrm{~mL}$ of fixative (3:1). This was repeated with two more re-suspensions in fixative.

\section{Slide preparation}

The method to prepare the slides was as described by Henegariu et al. (2001). In brief, $25-35 \mu \mathrm{L}$ of cell suspension was placed on the slide. As the fixative gradually evaporated, the surface of the slide became grainy (cells visible). At that moment, the slide was placed face down into the steam above a hot water bath $\left(75^{\circ} \mathrm{C}\right.$ or more) for 1-3 s, then dried by placing the slide on a metal plate suspended above the bath.

\section{Fluorescent in situ Hybridization - FISH}

This method was adapted from Finnon et al. (1995) and Hone et al. (2005). In brief, whole chromosome probes for \#2 and \#3 (Cambio) were hybridized onto the metaphases. The \#2 chromosomes were painted green with FITC (isothiocyanate) and the \#3 red with Texas Red. The metaphases were then counterstained with DAPI (4',6-diamidino-2-phenylindole) in Vectashield antifade mountant.

\section{Image Processing}

The analysis of metaphases detected by the FISH was performed using the fluorescence Zeiss microscopy with the triple band excitation: DAPIFITC-Texas Red, which incorporated an excitation filter with a narrow bandpass window in the ultraviolet (395 to 410 nanometers), blue (490 to 505 nanometers), and green (560 to 580 nanometers) spectral regions; with a digital camera (Axiocam $\mathrm{mRm}$ monochrome) with a resolution of 1388 x 1040 pixels and software ISIS (Metasystems, GmbH, Altlussheim, Germany). The members of chromosome pairs \#2 and \#3 in each cell were designated as ' $a$ ' or ' $b$ ' arbitrarily and their lengths were measured using the tools of the software ISIS (Metasystems, GmbH, Altlussheim, Germany), in 50 metaphases for each colcemid addition time. For this, the end of the chromosome was marked by the mouse, and a line was drawn along the chromosome to the other end. With this, the computer software converted the number of pixels covered to the length of the chromosome in micrometers. Gray scale images were generated by the Microsoft Paint (Niemeijer et al. 2004) in order to obtain a high (constant) threshold to better maintain the details of the chromosomes on a white background. With this, chromosomes \#2 became darker and chromosomes \#3 became clearer. The averages and standard errors of the chromosome lengths were calculated based on the measurements in 50 cells on the same slide.

\section{Chromosome length changes}

To determine whether the length changes of the painted chromosomes \#2 and \#3 induced by colcemid had a deterministic chaotic behavior, time series of length change were created using the 
following method modified from Lu et al. (1998) was employed. Supposing the length change of the jth chromosome of the ith cell in the $3 \mathrm{~h}$ colcemid exposure treatment was $\mathrm{L}_{3}(\mathrm{i}, \mathrm{j})$, and that of the corresponding data of the $k t h$ cell in the $48 \mathrm{~h}$ Colcemid exposure was $\mathrm{L}_{48}(\mathrm{k}, \mathrm{j})$, the difference between them was expressed as:

$$
\Delta \mathrm{L}_{3-48}(\mathrm{i}, \mathrm{j}, \mathrm{k})=\mathrm{L}_{3}(\mathrm{i}, \mathrm{j})-\mathrm{L}_{48}(\mathrm{k}, \mathrm{j})
$$

Where $i, k=1,2,3, \ldots 50 ; j=2 a ; 2 b ; 3 a$ or $3 b$. Consider $\Delta \mathrm{L}_{3-48}(1,2 \mathrm{a}, 1)$ as an example, where $\mathrm{i}=$ $\mathrm{k}=1$ and $\mathrm{j}=2 \mathrm{a}$, which meant a length difference between the chromosome $2 \mathrm{a}$ of the first cell in $3 \mathrm{~h}$ colcemid exposure, and of $2 \mathrm{a}$ of the first cell in 48 $\mathrm{h}$ colcemid exposure. One continues the procedure, $\Delta \mathrm{L}_{3-48}(2,2 \mathrm{a}, 1)=\mathrm{L}_{3}(2 \mathrm{a}, 2)-\mathrm{L}_{48}(2 \mathrm{a}, 1)$ according to equation $\{1\}$, which is checking for a length difference between chromosome $2 \mathrm{a}$ of the second cell in $3 \mathrm{~h}$ Colcemid, and of $2 \mathrm{a}$ of the first cell in $48 \mathrm{~h}$ Colcemid. The procedure continues until one reaches 2,500 possible combinations for one chromosome, and 10,000 combinations for the tow pairs $(2 a, 2 b, 3 a, 3 b)$. This first time series of length change was named " $\mathrm{A}$ ".

In order to evaluate the power of this method, other two time series were performed. The first, for each 50 differences, a shuffle was realized in the fixed column $(\mathrm{k} 1, \mathrm{k} 2, \mathrm{k} 3 \ldots, \mathrm{k} 50)$ in order to avoid a 'forced' repetition of the pattern of the graphic forced by the method and not as a result of the experiment. This time series was named " $\mathrm{B}$ ". At last, all the data were shuffled and named "surrogated" data, in order to confirm the randomness when the entire series was shuffled. This time series was named " $C$ ".

A curve could be plotted, with $i, k$ on the horizontal axis ( $\mathrm{i}, \mathrm{k}=1,2,3 \ldots 2,500)$, and length differences (in $\mu \mathrm{m}$ ), as calculated by the equation $\{1\}$ on the vertical axis. These combinations provided the time series representing the length change of chromosomes during the mitosis and the influence of colcemid in this dynamic process (Fig.1). Aiming to investigate the dynamics of these time series, the DFA algorithm was performed as described by Peng et al. (1994; 1995). The series was integrated and divided into boxes of equal length (n). In each box of length $n$, a least-square line was fitted to the data representing the trend in that box. The $y$ coordinate of the straight line segments was denoted by $\mathrm{yn}(\mathrm{k})$. Computing DFA was performed according to equation 2 .

$$
F(n)=\sqrt{\frac{1}{N} \sum[y(k)-y n(k)]^{2}}
$$

This computation was repeated over all the time scales (box sizes) to provide a relationship between the average root-mean fluctuation function $\mathrm{F}(\mathrm{n})$ and the box size $\mathrm{n}$ (i.e. the number of length changes in a box which was the size of the window of observation). Typically, $\mathrm{F}(\mathrm{n})$ would increase with box size $\mathrm{n}$. A linear relationship on a double log plot indicated the presence of scaling. Under such conditions, the fluctuations could be characterized by a scaling exponent alpha $(\alpha)$, the slope of the line relating $\log F(n)$ to $\log n: F(n) ~ ~$ $\mathrm{n}^{\alpha}$ (Fig. 3). In order to provide a more accurate estimate of $\mathrm{F}(\mathrm{n})$, the initial size of $\mathrm{n}$ was 10 , increasing by 10 reaching 2,500 points (10:10:2,500). If $\alpha=0.5$, the series was the result of a random event; $\alpha>0.5$ indicated the persistent long-range correlations. The other values were: $\alpha$ $=1$ corresponded to $1 / \mathrm{f}$ noise (very rough landscape); the $\alpha \geq 1$ - correlations existed but ceased to be of a power-law form or a random walk-like fluctuation; and finally, $\alpha=1.5-$ Brown noise; the integration of white noise (very smooth landscape) (Peng et al. 1995).

\section{RESULTS}

The earlier addition of colcemid into the cultures induced considerably more condensation of the measured chromosomes, \#2 and \#3 (Fig. 1-B). From analyzing the 50 metaphases, the average length of chromosomes \#2 and \#3 for $3 \mathrm{~h}$ exposure to colcemid were $9.1950 \pm 0.2991$ and $7.9040 \pm$ $0.2400 \mu \mathrm{m}$, respectively. For $48 \mathrm{~h}$ exposure to colcemid, the lengths of chromosomes \#2 and \#3 reduced to $6.3540 \pm 0.2082$ and $5.7950 \pm 0.1933$ $\mu \mathrm{m}$, respectively $(\mathrm{p}<0.05)$.

In order to study the dynamics of chromosome condensation to identify if this process was random or deterministic chaotic, the differences of lengths of chromosomes 2 and 3 between the two stages were obtained. Figure 2 shows one time series obtained for chromosome $2 \mathrm{a}$.

A fitted line of $\log F(n)-\log n$ with $\log n$ as horizontal axis and $\log F(n)$ as vertical axis was obtained, and the slope of this line was the value of the scaling exponent $(\alpha)$ for the time series of length differences of chromosome $2 \mathrm{a}$ (Fig. 3). The same procedure was repeated for chromosomes $2 b$, $3 a$ and $3 b$. 


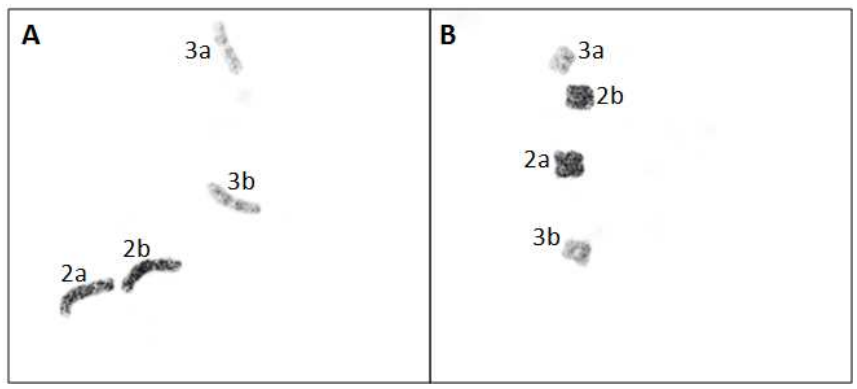

Figure 1 - Chromosomes \#2 and \#3 with different durations of Colcemid exposure. (A) final 3 h; (B) final 48 h (\#2 darker; \#3 clearer).

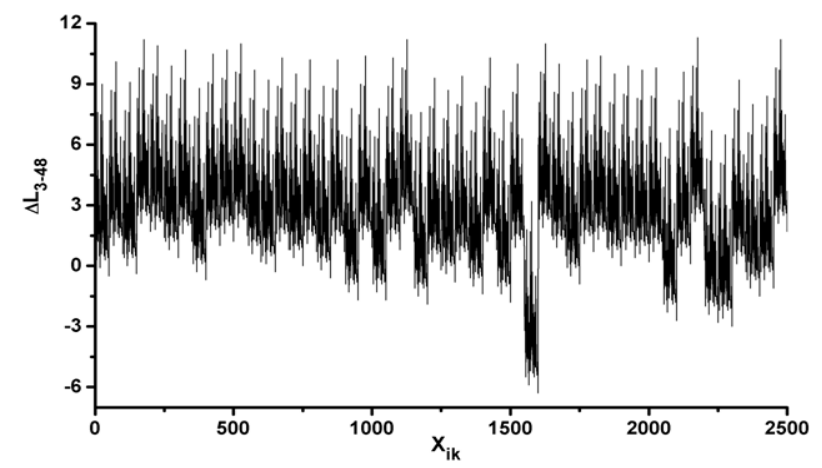

Figure 2 - A plotting wave of 2,500 variations. Xik: cell series order $(1,2, \ldots$ 2,500); $\Delta \mathrm{L} 3-48$ : the difference of chromosome \#2a lengths from $3 \mathrm{~h}$ and $48 \mathrm{~h}$ of Colcemid treatment.

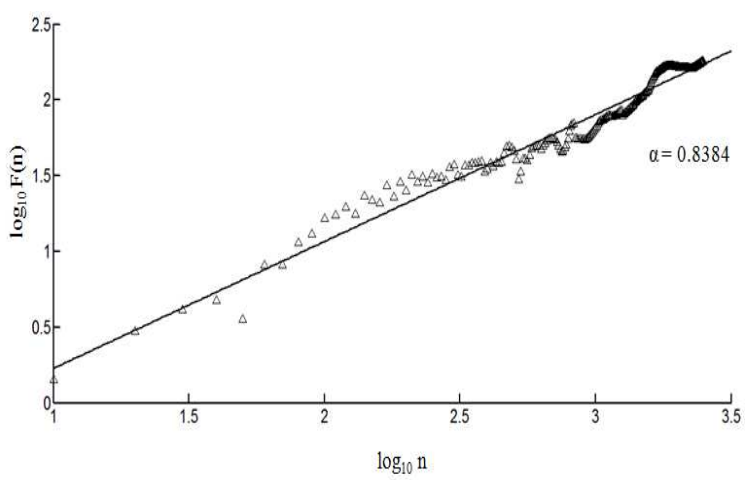

Figure 3 - Plot of $\log 10 \mathrm{~F}(\mathrm{n})$ vs $\log 10 \mathrm{n}$ for 2,500 length changes of chromosomes \#2a from $3 \mathrm{~h}$ and $48 \mathrm{~h}$ of Colcemid treatment, with scaling exponent $\alpha=0.8384$ (see description of DFA computation in text).

In Figure 4, it is possible to visualize a magnified part of the curve for only 150 length differences for the data non-shuffled (series ' $\mathrm{A}$ '), for shuffling the column in each 50 length changes (series 'B'), and for the surrogated data (series ' $\mathrm{C}$ '). In $\mathrm{A}$, it is possible to see the identical pattern of the graphic in each 50 length changes (I, I, I). By contrast, this tendency was lost in B (I, II, III) and in C (I, II, 
III). The alpha $(\alpha)$ values in A and B were very close to each other $(0.8384$ and 0.8334 , respectively), demonstrating that the event was nonrandom or deterministic chaotic, and this was not caused by fixing the column of subtracted values, because even when the column was shuffled, the value of $\alpha$ remained characteristic of a deterministic chaotic phenomenon. On the other hand, the value of $\alpha$, after surrogating all the 2,500 values of length changes, decreased to 0.5516 , correspondent to random events and demonstrating again the power of the method.

Series B confirmed that the deterministic chaotic behavior of the chromosome condensation was not forced by the repetition of the pattern observed in A, because $\alpha$ value continue greater than 0.5 .
Series C confirmed that the surrogated data had $\alpha$ value around 0.5 as expected by the randomization of the entire data, demonstrating the power of the DFA method in identifying the long-range correlations and the presence of randomness in time series.

Table 1 shows the values of $\alpha$ and standard errors for all the time series (series A, B and C). The average $\alpha$ when the data were not shuffled was $0.8550 \pm 0.0192(n=4)(A)$; when the column of subtracted column was shuffled in each 50 length changes to avoid the repetition of the patter of the graphic, the average $\alpha$ was $0.8522 \pm 0.0214$ (B); and when all data were shuffled (surrogated data), the average $\alpha$ was $0.5098 \pm 0.0549$ (C).

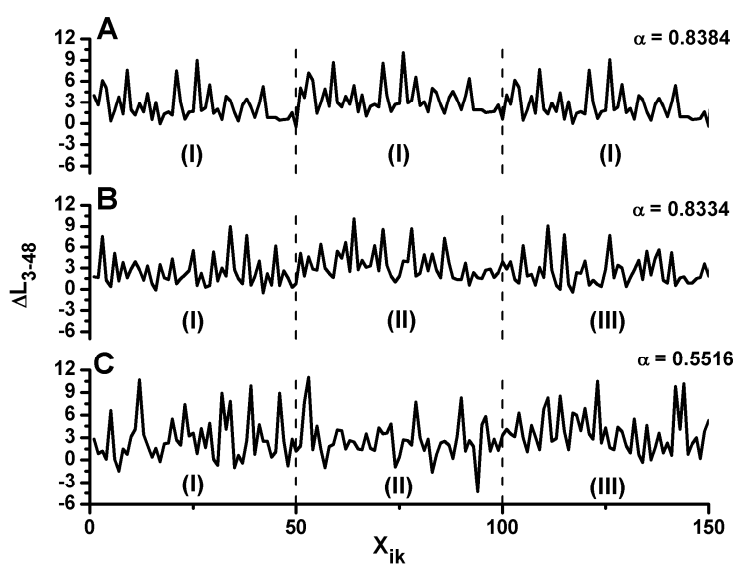

Figure 4 - A plotting wave of 150 variations of chromosome \#2a. Xik: cell series order $(1,2, \ldots$ 2,500); $\Delta$ L3-48: the difference of chromosome lengths. Series: A (non-shuffled); B (shuffled); and C (surrogated). Roman numbers refers to the pattern of the graphic in each 50 differences; $\alpha$ value is the scaling exponent from DFA.

Table 1 - Scaling exponents $(\alpha)$ for all length changes of chromosomes \#2a, 2b, 3a and 3b, from $3 \mathrm{~h}$ and $48 \mathrm{~h}$ of Colcemid treatment.

\begin{tabular}{cccc}
\hline Series & \multicolumn{3}{c}{ Alpha $(\boldsymbol{\alpha})$ exponent from DFA analysis } \\
\hline 2a & Non-shuffling column & Shuffling column & Shuffling all data \\
2b & 0.8384 & 0.8334 & 0.5516 \\
3a & 0.8595 & 0.8678 & 0.4299 \\
3b & 0.8418 & 0.8342 & 0.5192 \\
Average \pm SD $^{*}$ & 0.8802 & 0.8735 & 0.5383 \\
\hline
\end{tabular}

$* \mathrm{SD}=$ standard deviation

\section{DISCUSSION}

It is well known that the genetic material in the nuclei are gradually coiled and folded from a linear DNA duplex into the chromosomes spatial structures, which assumes functions in a non-linear way. Understanding how chromosomes condense can provide new insights into the complex 
relationship between the chromatin structure, gene expression and the functional state of the cell (Lieberman-Aiden et al. 2009). Liberman-Aiden et al. (2009) described a non-equilibrium conformation of chromatin, which permited its condensation and decondensation, as a "fractal globule". Fractal globules are an attractive structure for chromatin because they could facilitate the condensation and decondensation during gene activation and gene expression or during the cell cycle, with an ideal optimization of space in order to organize a long molecule of DNA into a coiled complex structure of just a few micrometers (Liebovitch 1998; Lieberman-Aiden et al. 2009). This could be useful information for better understanding the dynamics of living cells, and possibly predicting the disorders induced by the chemicals, biological or physicals agents, as already has been done for the prognostics of some types of cancer (Bendin et al. 2010; Einstein et al. 1998).

Chromosome configuration and dynamics in a population of cells are helping to develop new physics-based models. Analysis techniques will be important to make full use of these data and to better understand the mechanisms underlying nuclear organization (Zimmer and Fabre 2010). In yeast, some information on chromatin condensation has been derived from the FISH experiments, in which spatial distances were measured between the loci located at various genomic intervals along chromosomes. A recent reanalysis of in vivo measurements for the chromosome 3 supported a structure of $\sim 1.2-3.6$ nucleosomes per $11 \mathrm{~nm}$. This variability might be explained by the histone modification and variations in the length of the sequence of linker DNA between the consecutives nucleosomes, which were expected to modulate the nucleosome occupancy and chromatin packing (Zimmer and Fabre 2010).

The effect of highly chromosome condensation induced by colcemid concentration has already been discussed elsewhere (Fernandes et al. 2008b). In the present paper, the data demonstrated that DFA was a powerful computational method to determine that the phenomenon of high chromosome condensation induced by the prolonged treatment with colcemid was a deterministic chaotic or nonrandom event (Figure 4-A). This was verified by shuffling the values of fixed column in each 50 length changes (series B), which maintained the scaling exponent $\alpha$ about the same, confirming that the event was deterministic chaotic and not forced by the method. Also, when all data were shuffled (surrogated data, C), the $\alpha$ decreased to 0.5 , characteristic of random events, confirming the reliability of DFA method. The results presented here indicated that the dynamical process of chromosome condensation was nonlinear and deterministic chaotic, probably due to the fractal feature of chromosome packing.

\section{CONCLUSION}

This work was designed to investigate the effects of colcemid on the dynamical process of chromosome condensation. When colcemid was put into lymphocyte cultures for a longer time (48 $h$ ), it induced the phenomenon of excessive chromosome condensation, and the dynamic of this process was deterministic chaotic with longterm correlation with values of $0.5<\alpha \leq 1.0$, characteristic of the nonlinear property of human chromatin. This could be due to the toxic action on the mitotic spindle or in some manner to the proteins of chromosomes, but these remained unknown and poorly described in the literature. Further work is needed to elucidate the molecular causes of such phenomenon.

\section{ACKNOWLEDGEMENTS}

The authors would like to thank Dr. David Lloyd (Health Protection Agency, UK), for English reviews, comments and suggestions; the Conselho Nacional de Desenvolvimento Científico e Tecnológico (CNPq, Brazil), Coordenação de Aperfeiçoamento de Pessoal de Nível Superior (CAPES, Brazil), and Fundação de Amparo à Ciência e Tecnologia do Estado de Pernambuco (FACEPE, Brazil), for supporting this work.

\section{REFERENCES}

Amaral A, Fernandes TS, Cavalcanti MB. Bioindicators in Radiation Protection. Braz Arch Biol Technol. 2008; 51: 91-96.

Bendin V, Adam RL, Sá BCS, Landman G, Metze K. Fractal dimension of chromatin is an independent prognostic factor for survival in melanoma. $B M C$ Cancer. 2010; 19: 1-6. 
Edwards AA, Lindholm C, Darroudi F, Stephan G, Romm H, Barquinero J, et al. Review of translocations detected by FISH for retrospective biological dosimetry applications. Radiat Prot Dos. 2005; 113: 396-402.

Einstein AJ, Wu HS, Gil J. Self-afinity and lacunarity of chromatin texture in benign and malignant breast epithelial cell nuclei. Phy Rev Let. 1998; 80: 397-400.

Fernandes TS, Lloyd DC, Amaral A. Biodosimetry for dose assessment of partial-body exposure: a methodological improvement. Braz Arch Biol Technol. 2008; 51: 97-102.

Finnon P, Lloyd DC, Edwards AA. Fluorescence in situ hybridization detection of chromosomal aberrations in human lymphocytes: applicability to biological dosimetry. Int J Radiat Biol. 1995; 68: 429-435.

Havlin S, Buldyrev SV, Bunde A, Goldberger AL, Ivanov PCh, Peng CK, et al. Scaling in nature: from DNA through heartbeats to weather. Phys A. 1999; 273: 46-49.

Hayata I, Kajima J, Okabe N. Distinction of metaphases in the first cell cycle for automated system in radiation dosimetry. Radiat Phys Chem. 1992; 39: 517-520.

Henegariu O, Heerema NA, Wright LL, Bray-Ward P, Ward DC, Vance GH. Improvements in cytogenetic slide preparations: controlled chromosome spreading, chemical aging and gradual denaturing. Cytometry. 2001; 43: 101-109.

Hone PA, Edwards AA, Lloyd DC, Moquet JE. The yield of radiation-induced chromosomal aberrations in first division human lymphocytes depends on the culture time. Int J Radiat Biol. 2005; 81: 523-529.

International Atomic Energy Agency (2001) Cytogenetic analysis for radiation dose assessment. Vienna. (IAEA Technical Report Series; 405).

Kanda R, Jiang T, Hayata I, Kobayashi S. Effects of Colcemid concentration on chromosome aberration analysis in human lymphocytes. J Radiat Res. 1994; 35: 41-47.

Kunicki ACB, Oliveira AJ, Mendonça MBM, Barbosa CTF, Nogueira RA. Can the fractal dimension be applied for the early diagnosis of non-proliferative diabetic retinopathy? Braz J Med Biol Res. 2009; 42: 930-934.

Lieberman-Aiden E, Berkum NL, William L, Imakaev M, Ragoczy T, Telling A, et al. Comprehensive mapping of long-range interactions reveals folding principles of the human genome. Science. 2009; 326: 289-293.

Liebovitch LS. Fractals and chaos simplified for life sciences. New York: Oxford University Press; 1998.

Lu R, Wang Z, Tang Y, Qui N. Fractal behavior of length change in human mitotic chromosome. $J$ Tongji Med. 1998; 4: 201-203.

Mandelbrot BB. The Fractal Geometry of Nature. 2nd. ed. New York: Ed. Freeman; 1983.
Meleiro LAC, Costa AC, Filho RM. Non-linear multivariable prediticve control of an alcoholic fermentation process using functional link networks. Braz Arch Biol Technol. 2005; 48: 7-18.

Niemeijer $M$, Staal J, van Ginneken B, Loog $M$, Abramoff M. Comparative study of retinal vessel segmentation methods on a new publicly available database. SPIE Med Imag. 2004; 5370: 648-656.

Peng CK, Buldyrev SV, Havlin S, Simons M, Stanley HE, Goldberger AL. Mosaic organization of DNA nucleotides. Phys Rev E. 1994; 49: 1685-1689.

Peng CK, Havlin S, Stanley HE, Goldberger AL. Quantification of scaling exponents and crossover phenomena in non-stationary heartbeat time series. Am Inst Phys. 1995; 5: 82-87.

Sasaki MS, Kobayashi K, Hiedas K, Yamada T, Ejima $\mathrm{Y}$, Maezawa $\mathrm{H}$, et al. Induction of chromosome aberrations in human lymphocytes by monochromatic X-rays of quantum energy between 4.8 and $14.6 \mathrm{keV}$. Int J Radiat Biol. 1989; 56: 975-988.

Schwartz EL. Antivascular actions of microtubulebinding drugs. Clin Can Res. 2006; 15: 2594-2601.

Souza GM, Oliveira RF, Cardoso VJM. Tempora dynamics of stomatal condutance of plants under water deficit: can homeostasis be improved by more complex dynamics? Braz Arch Biol Technol. 2004; 47: 423-431.

Stam CJ. Nonlinear dynamical analysis of EEG and MEG: Review of an emerging field. Clin Neurophysiol. 2005; 116: 2266-2301.

Stanley HE, Afanasyev V, Amaral LAN, Buldyrev SV, Goldberger AL, Havlin S, et al. Anomalous fluctuations in the dynamics of complex systems: from DNA and physiology econophysics. Phys A. 1996; 224: 302-321.

Tanizawa H, Iwasaki O, Tanaka A, Capizzi JR, Wickramasinghe $\mathrm{P}$, Lee $\mathrm{M}$, et al. Mapping of longrange associations throughout the fission yeast genome reveals global genome organization linked to transcriptional regulation. Nucleic Acid Res. 2010; 38: 8164-8177.

Wang SC, Li PC, Tseng HC. Long range correlation and possible electron conduction through DNA sequences. Phys A. 2008; 387: 5159-5168.

Zimmer C, Fabre E. Principles of chromosomal organization: lessons from yeast. JCB: Review. 2010; 7: $723-733$.

Received: October 06, 2011; Revised: December 01, 2011; Accepted: August 20, 2012. 